\title{
La relevancia de los amicus curiae en los arbitrajes internacionales de inversión pos COVID-19 en materia de salud pública
}

\author{
Christian Carbajal Valenzuela* \\ Yolanda Mendoza Neyra*
}

\section{RESUMEN}

A fin de reducir la propagación del virus COVID-19, los Estados vienen adoptando diversas medidas para resguardar la salud pública y los demás intereses de sus poblaciones, acciones que no están exentas de críticas, algunas serán consideradas necesarias y razonables, adoptadas de conformidad con las facultades regulatorias estatales previstas por el derecho internacional. Otras, en cambio, por ser arbitrarias, desproporcionales y discriminatorias, podrán ser entendidas como violatorias del derecho internacional de las inversiones y de específicos estándares contenidos en los tratados de inversión. Probablemente, en relación con muchas de esas medidas, las voces más discordantes serán de los inversores extranjeros que considerarán sus intereses severamente perjudicados.

Por esta situación de colisión entre regímenes jurídicos e intereses — protección de la salud pública vs protección de la inversión extranjera-, este artículo explora cómo los amicus curiae, en momentos de emergencia como el que estamos viviendo, resultarán de especial

\footnotetext{
* Abogado por la Pontificia Universidad Católica del Perú (PUCP). Magíster en Derecho Internacional Económico por la Universidad de Warwick, Inglaterra. Asesor legal en arbitrajes internacionales CIADI y CCI. Profesor de los cursos Derecho Comercial Internacional y Arbitraje Internacional de Inversiones en Universidades del Perú y Brasil. Socio de Wöss \& Partners S.C. (W\&P) en México y Washington D.C. y de Braz Campos Advogados en São Paulo y Curitiba, Brasil. Director de Arbitraje de la Cámara de Arbitraje y Mediación de Santa Catarina (CAMESC), Brasil. Correo electrónico: ccarbajal@woessetpartners.com christian@brazcampos.com.br LinkedIn: linkedin.com/ in/christianarmandocarbajal/

(iD) https:///orcid.org/0000-0002-2918-5550

* Abogada por la Universidad de Lima, con Segunda Especialidad en Derecho Internacional Público por la Pontificia Universidad Católica del Perú. Correo electrónico: a20183609@pucp.edu.pe

(iD) https://orcid.org/0000-0002-3025-5018
} 
relevancia para la emisión de decisiones razonables por los tribunales en los próximos arbitrajes internacionales de inversión referidos a salud pública. De consolidarse la participación adecuada de amicus curiae en procedimientos con claro interés público involucrado, será un elemento que contribuirá de manera fundamental en la implementación de mecanismos de transparencia en el sistema de solución de controversias entre inversionistas y Estados, absolutamente necesario para su legitimidad.

Palabras clave: amicus curiae, salud pública, COVID-19, derecho internacional de las inversiones, derecho global de la salud, arbitraje internacional de inversiones.

The relevance of Amicus Curiae in the post COVID-19 international investment arbitrations related to public health

\section{Abstract}

To reduce the spread of the COVID-19 virus, States have been taking various measures to safeguard public health and other interests of their populations, actions that are not exempt from criticism, some will be considered necessary and reasonable, adopted in accordance with the state regulatory powers provided for by International Law. Others, on the other hand, because they might be considered arbitrary, disproportionate, and discriminatory, will be understood as violating International Investment Law and specific standards contained in Investment Treaties. Probably, in relation to many of these measures, the most discordant voices will be those of foreign investors, who may consider their interests severely harmed.

Because of this situation of collision between legal regimes and interests - protection of public health vs. protection of foreign investment-, this article explores how amicus curiae, in times of emergency such as the one we are experiencing, will be of particular relevance to the issuance of reasonable decisions by arbitral tribunals in the forthcoming international investment arbitrations concerning public health. If the appropriate participation of amicus curiae in procedures with a clear public interest involved is consolidated, it will be an element that will contribute fundamentally to the implementation of transparency mechanisms in the investor-state dispute settlement system, which is absolutely necessary for its legitimacy.

Keywords: Amicus Curiae, public health, COVID-19, International Investment Law, Global Health Law, International Investment Arbitration.

\section{Introducción}

No cabe duda de que durante los últimos quince años el sistema de solución de controversias entre inversores y Estados (SSCIE) ha buscado responder a diversas críticas que han afectado su legitimidad. En gran medida, la negociación y aprobación de nuevas generaciones de tratados de inversiones, y las reformas ya realizadas o en curso, a las reglas aplicables a los arbitrajes internacionales de inversión (AII), han permitido un cierto nivel de renovación en el SSCIE, a pesar de que algunos detractores proponen su eliminación. A pesar de estos cambios, el SSCIE es aun 
potencialmente frágil, ya que subsisten problemas sistemáticos en su actual diseño, que aún no han sido totalmente corregidos en el seno de la Comisión de las Naciones Unidas para el Derecho Mercantil Internacional (CNUDMI) o en otros foros, sea a nivel multilateral o bilateral.

Uno de los problemas que el SSCIE enfrenta es su falta de transparencia (CNUDMI, $2017, \$ 20)$. Aunque consideramos que esta última es actualmente relativa, pues se ha buscado subsanar dicha carencia con la incorporación de mecanismos de transparencia como, por ejemplo, la participación de terceras partes no contendientes, que en la práctica arbitral de inversiones está referida a la participación de amicus curiae (Saei, 2017, p. 247). Si bien este es un instrumento del derecho procesal —el mismo se encuentra regulado expresa y tácitamente en el SSCIE—, aún no ha tenido especial relevancia para algunos tribunales arbitrales de inversión al momento de resolver las disputas (Born y Forrest, 2019, p. 648), o simplemente han sido rechazados al considerarse que las respectivas solicitudes no han cumplido algún requisito de admisibilidad.

Debido a la actual crisis sanitaria mundial que estamos viviendo, es altamente probable que el SSCIE enfrente nuevos retos, que podrían, en el mejor de los casos, acelerar un mayor consenso global para lograr una satisfactoria reforma del sistema. Tal reto estaría relacionado con el impacto que tendrían los amicus curiae sobre salud pública en las próximas decisiones de los tribunales arbitrales, cuyos argumentos adicionales y perspectivas particulares — principalmente aquellos con sólidos fundamentos científicos—, serán determinantes para resolver en forma más adecuada los conflictos de inversiones extranjeras que involucren dicho interés público. Si los argumentos de estos amicus curiae son tomados en cuenta por los tribunales arbitrales como corresponde, esta práctica sentará las bases para una real apertura y transparencia general de los AII. Desde nuestra perspectiva, los amicus curiae sobre salud pública, esta vez, gozarán de mayor consideración y tendrán un impacto más relevante en los AII.

Por lo expuesto, el propósito de esta investigación académica, además de examinar si existe una verdadera protección del interés de la salud pública en los AII, es analizar de manera más cercana los posibles alcances que tendrían los amicus curiae en las demandas de arbitraje relacionadas con el COVID-19. Por estos motivos, hemos optado por desarrollar el presente artículo de la siguiente forma: en la Sección 2 explicaremos las justificaciones que tienen mayor consenso en el ordenamiento internacional para aceptar los amicus curiae en los AII; en la Sección 3 indicaremos los instrumentos internacionales de la región sobre inversión, con disposiciones expresas o tácitas relativas a la presentación de amicus curiae; en la Sección 4 describiremos cómo los principales mecanismos procedimentales regulan los amicus curiae en sus 
Reglamentos de Arbitraje; y, finalmente, en la Sección 5, nos enfocaremos en el análisis del impacto de los amicus curiae sobre la salud pública y los posibles efectos que tendrán en los próximos AII que se iniciarán a causa del COVID-19.

\section{Las justificaciones de los amicus curiae en los arbitrajes internacionales de inversión}

La incorporación de los amicus curiae a la estructura procedimental de los AII no fue una iniciativa de los Estados. En principio, fueron los tribunales arbitrales internacionales que, frente a conflictos muy complejos, voluntariamente aceptaron las opiniones técnicas, jurídicas y científicas de partes no contendientes, bajo la institución del derecho procesal de amicus curiae. Esta tercera parte no contendiente que apoya a los tribunales de manera desinteresada, y que tiene sus orígenes en el derecho romano, paradójicamente fue introducida en los procesos jurídicos internacionales por Estados que se encuentran regidos por el common law (Kent y Trinidad, 2016, pp. 1083-1086).

Como ha sido mencionado, el uso de amicus curiae en el SSCIE es una de las respuestas que se adoptaron para recuperar la imagen pública dañada del sistema. Los AII casi siempre comprenden asuntos que no tienen únicamente un origen privado, hay en la mayoría de los casos un interés público también involucrado, precisamente porque una de las partes del conflicto es el Estado y porque están involucradas en el caso facultades regulatorias. Por ello la especial relevancia del amicus curiae en este contexto.

Debido a la naturaleza pública de los Estados, en la práctica procedimental, la participación de terceras partes no contendientes mediante amicus curiae pasa a ser un asunto trascendental, ya que, por un lado, los AII se refieren a cuestiones que implican decisiones estatales adoptadas con el objetivo de proteger el interés público. Por otro lado, laudos de inversión expedidos sin que los tribunales arbitrales hayan evaluado adecuadamente ese interés público y/o no reparen en el valor de las políticas públicas nacionales e internacionales, además de mantener las deficiencias del SSCIE, perjudicarán el bienestar general de los nacionales de los Estados demandados. De allí que, en este contexto, los amicus curiae resulten relevantes para lograr la recuperación de la legitimidad y la transparencia del SSCIE.

Tras esta breve explicación referida a la introducción de los amicus curiae en los AII, a continuación desarrollaremos las justificaciones que existen para su uso: el interés público, la política pública internacional, y la transparencia. 


\subsection{El interés público}

En vista de la conexión directa entre las decisiones finales de los AII y los efectos que tendrán sobre el bienestar y los intereses de terceros, especialmente si las decisiones tienen impactos perjudiciales sobre ellos, el SSCIE reconoció el derecho a participar de terceras partes no contendientes en sus procedimientos arbitrales. Aunque con limitaciones para su ejercicio, ya que además de cumplir con ciertos requisitos para su presentación, queda a la discrecionalidad de los tribunales arbitrales emplear las opiniones técnicas como sustento de sus decisiones, la participación de los amicus curiae configura un paso importante del SSCIE, en su esfuerzo por reconciliarse con parte de la comunidad internacional, que exige su reforma radical o abolición.

Precisamos que, el interés público y/o los intereses públicos a los cuales nos referimos, son intereses de la sociedad civil o de los terceros que pueden verse afectados por las demandas de los inversionistas extranjeros o por los actos u omisiones estatales, que guardan una relación esencial con aspectos de la soberanía de los Estados y el bienestar de sus nacionales o con bienes comunes de la humanidad, tales como los derechos humanos (Butler, 2019, pp. 147 y 148). Por ello, resulta lógico que en caso se perciba una afectación directa o indirecta al interés público por la decisión de un tribunal internacional, se necesite una defensa especial o una representación más amplia que la ofrecida por el propio Estado demandado. El interés público que pueda estar involucrado en los AII surge de las legislaciones nacionales o de los propios tratados que buscan promover políticas públicas económicas, laborales, ambientales, energéticas, mineras, alimentarias, educacionales, de servicios públicos, salud pública, entre otros y también de la protección de bienes elementales y universales, como son los derechos humanos. Para esclarecer o informar a los tribunales arbitrales sobre ciertos aspectos técnicos complejos o relevantes, de interés general, alegados u omitidos por las partes, consideramos que los amicus curiae tienen un rol clave en dichos procedimientos arbitrales.

Hasta el momento, además de ser la principal forma en que las preocupaciones de los terceros con intereses genuinos pueden hacerse visibles en la esfera de las inversiones extranjeras, los amicus curiae facilitan a los tribunales arbitrales la identificación del interés público que podría potencialmente ser afectado con sus decisiones. Para distinguir a quiénes debemos reconocer como los más afectados en una controversia sobre inversiones extranjeras, se debe analizar los factores alegados por los inversionistas demandantes que, según sus apreciaciones, causan la vulneración de sus derechos protegidos por los tratados de inversión, así como los argumentos de los Estados referidos al ejercicio de facultades regulatorias. 
Es absolutamente necesario que los AII garanticen transparencia procesal ya que, reiteramos, la solución podría afectar negativamente los intereses de toda una sociedad (Saei, 2017, pp. 272 y 273). Cabe recordar que fue en base al interés público que los tribunales arbitrales autorizaron inicialmente la intervención de terceras partes no contendientes bajo la figura de los amicus curiae en los AII, a pesar de la falta de regulación. Para ejemplificar estas incorporaciones pioneras en los procedimientos arbitrales del SSCIE, nos remitimos a las decisiones de los tribunales sobre peticiones de terceras personas para intervenir como amicus curiae del 15 de enero de 2001, en el caso CNUDMI Methanex Corporation c. Estados Unidos de América (\$29-31), y del 17 de octubre de 2001, en el caso CIADI United Parcel Service of America Inc. (UPS) c. Canadá (\$ 61), así como la orden en respuesta a una petición de cinco organizaciones no gubernamentales de permiso para hacer una presentación de amicus curiae, del 12 de febrero de 2007, en el caso CIADI Suez, Sociedad General de Aguas de Barcelona, S.A. y Vivendi Universal, S.A. c. Argentina ( $\$ 10$ y 11).

\subsection{La política pública internacional}

Entendida también como política pública transnacional, su aplicación es de especial relevancia en los AII, puesto que, mediante ella, de un lado, se evalúa la conducta de las partes para determinar la jurisdicción de los tribunales arbitrales y la admisibilidad de la demanda. De otro lado, se legitiman los laudos arbitrales, evitando su anulación o inejecutabilidad en instancias nacionales. Asimismo, esta segunda justificación del uso de los amicus curiae, es utilizada para aplicar y mantener principios y valores universales reconocidos por la comunidad internacional (De Brabandere, 2020, p. 852). Por ejemplo, esos principios y valores están recogidos en el preámbulo de la Carta de las Naciones Unidas, las normas ius cogens, la costumbre internacional, y en los altos estándares morales, éticos y de justicia aceptados por todas las naciones, tales como la prohibición de la corrupción, el terrorismo, el fraude, entre otros.

Al estar incluida en el derecho internacional, los tribunales arbitrales del SSCIE podrían considerarla como parte de las leyes aplicables para cada caso en concreto. Dado que el origen de esos tribunales se encuentra en las disposiciones de los tratados de inversión, en este nivel jurídico es indispensable que esos tribunales internacionales no omitan ese conjunto de principios y valores universales de naturaleza imperativa, descritas en el párrafo anterior.

Esa aplicación obligatoria permitirá que laudos arbitrales gocen de legitimidad y reconocimiento internacional, y no sean concebidos como instrumentos inconsistentes que ocasionen dańos irreparables o sean contrarios a las necesidades de los agentes no estatales. Por ello, resulta importante que la interpretación de los tribunales arbitrales 
sobre la política pública trasnacional esté acorde con el derecho internacional ya que, al estar regidos por este ordenamiento jurídico, frente a la complejidad de los regímenes jurídicos internacionales, será posible resolver los conflictos normativos (Saei, 2017, pp. 274 y 275).

Una de las formas en que se configura la política pública internacional en los arbitrajes internacionales de inversiones son los principios procesales, entre ellos el debido proceso (Artículo V de la Convención sobre el Reconocimiento y la Ejecución de las Sentencias Arbitrales Extranjeras de 1958). Además de implicar el trato equitativo entre las partes, mediante dicho principio se garantiza que los tribunales sean imparciales, razonables y justos. En este contexto, la importancia de los amicus curiae radica en que hace posible ampliar el derecho de defensa de las partes, ya que la asistencia brindada a los tribunales arbitrales con información adicional y útil para esclarecer o hacer saber de situaciones que comprendan algún interés público, a su vez contribuye a que tales tribunales tengan mayores elementos para vislumbrar — de cierta forma- la solución de los conflictos.

\subsection{La transparencia}

Parte de la actual crisis del SSCIE, está vinculada, en parte, con su falta de transparencia. Como mecanismo de solución y colaboración, se promovió la transparencia desde el contexto procesal, con la apertura responsable de los AII, permitiendo la participación legal de terceras partes no contendientes como los amicus curiae. Sin embargo, reiteramos que dicha transparencia aún es parcial (Maupin, 2013, pp. 156-162), además existe una resistencia significativa en algunos tribunales arbitrales, especialmente cuando debaten la afectación o no de los intereses públicos.

Consideramos que la transparencia, como elemento crucial y significativo para la legitimidad del SSCIE, no debe ser percibida como un factor contraproducente y peligroso para la autonomía de los tribunales arbitrales o que cause incompatibilidad con la confidencialidad en las disputas. Al contrario, la transparencia, por ser un valor propio del Estado de derecho (Butler, 2019, p. 148), fortalece la igualdad de los interesados y la credibilidad de las soluciones adoptadas por tribunales arbitrales. En cuanto a lo último, con una mayor transparencia mediante los amicus curiae, también se logra que el desempeńo de los árbitros internacionales de inversión sea aceptado con menores cuestionamientos, en la medida que otorga seguridad —al contar con conocimientos más sólidos e información relevante-, e independencia en la adopción de sus decisiones.

La inclusión de la transparencia con los amicus curiae en el derecho internacional de las inversiones, se reflejó en la adopción de disposiciones sobre requisitos de transpa- 
rencia procesal en los tratados de inversión, las modificaciones en 2006 al Convenio sobre Arreglo de Diferencias Relativas a Inversiones entre Estados y Nacionales de Otros Estados (en adelante Convenio CIADI), las Reglas de Arbitraje CIADI (en adelante Reglas CIADI), el Reglamento de Arbitraje del Mecanismo Complementario CIADI, y la adopción del Reglamento de la CNUDMI sobre la Transparencia en los Arbitrajes entre Inversionistas y Estados en el Marco de un Tratado de 2013 (en adelante Reglamento CNUDMI sobre Transparencia), que complementa las Reglas de Arbitraje CNUDMI. Asimismo, en este contexto, se aprobó un instrumento multilateral como la Convención de las Naciones Unidas sobre la Transparencia en los Arbitrajes entre Inversionistas y Estados en el Marco de un Tratado de 2014 conocida como Convención de Mauricio sobre Transparencia-, y que está en vigor desde 2017.

Mediante dicha Convención se dispone que tal Reglamento CNUDMI sobre Transparencia será aplicable a los arbitrajes internacionales de inversión, si los tratados de inversión por los que surgen fueron celebrados antes del 1 de abril de 2014, y además: i) si el inversionista demandante es de un Estado Parte que no haya formulado ninguna reserva sobre un determinado tratado de inversión; ii) si el Estado demandado como Estado Parte no haya formulado reserva del artículo 3.1a); y, iii) si el Estado demandado como Estado Parte no haya formulado reserva alguna del artículo 3.1, siempre y cuando el inversionista demandante no se oponga a la aplicación del mencionado Reglamento CNUDMI. En cuanto a las disposiciones generales de este último, las mismas que explicaremos con mayor profundidad en el punto 4 de esta investigación, señala, entre otros: i) que el ejercicio de las facultades discrecionales de los tribunales arbitrales tendrán en cuenta el interés público; ii) la admisión de escritos de terceros; iii) la publicación de los actos procedimentales, inclusive de oficio, pero con ciertas excepciones; $y$, iv) audiencias públicas con limitaciones.

\subsection{Los argumentos a favor y en contra sobre los amicus curiae}

Los cambios realizados en el SSCIE desde inicios de este siglo, para adecuarse a las nuevas exigencias de la comunidad internacional, si bien permiten que sea percibido como más responsable, eficiente y menos tradicional - sin afectar los objetivos y fines de su creación, como son la promoción de la inversión extranjera y del desarrollo económico-, siempre serán objeto de diversas críticas, favorables o negativas. Como es de esperarse, la implementación de los amicus curiae en el SSCIE no tendría que ser la excepción, más aún cuando los amici curiae no son parte contendiente en las disputas. En líneas generales, los argumentos que explican su contribución positiva o negativa al SSCIE, los hemos recogido y sintetizado, sin ningún orden en particular, en los siguientes párrafos. 
Además de promocionar la transparencia y la publicidad de los AII, como hemos señalado en los anteriores subpuntos, con la participación de los amici curiae se busca la protección de los intereses públicos, y brindar apoyo a los tribunales arbitrales con información transcendente, complementaria o distinta de los argumentos ofrecidos por las partes. Asimismo, los amicus curiae, en principio, permiten que se emitan laudos arbitrales de mayor calidad técnica y jurídica, en vista que los árbitros no son especialistas en todos los regímenes del derecho internacional ni en áreas sociales o científicas, defecto que les podría generar dificultades para tener un conocimiento amplio y actualizado para solucionar con efectividad las controversias. Gracias a los amicus curiae, de manera independiente, se informa a los tribunales arbitrales de inversión sobre las consecuencias y los impactos negativos que sus decisiones podrían ocasionar a los individuos ajenos al procedimiento arbitral —especialmente a las poblaciones de los Estados demandados-, si estas se emiten sin tener en consideración la política pública internacional o los intereses públicos afectados.

Por otro lado, los críticos de los amicus curiae señalan que estos serán contraproducentes y riesgosos para el SSCIE, puesto que aumentarán los costos de los procedimientos arbitrales, aumentará la duración de los procesos, eliminará la confidencialidad y la privacidad de los arbitrajes, así como la solución pacífica de las disputas. También podrían ser utilizados para afectar la autonomía de los tribunales arbitrales y la igualdad entre las partes, logrando la politización y desbalance del SSCIE, lo cual produciría profundos efectos contrarios a los esperados, tales como la deslegitimación del sistema y de los AII.

\section{El tratamiento de los amicus curiae en los instrumentos internacionales de inversión}

Para regular la representación de los intereses públicos que podrían verse afectados con las decisiones arbitrales, y a su vez introducir mecanismos que posibiliten la transparencia procesal y legal de los AII, los Estados han suscrito tratados de inversión que permiten la participación de terceras partes no contendientes en el SSCIE. Este respaldo estatal fue recogido por primera vez en los modelos de tratados bilaterales de inversión de Estados Unidos de América y de Canadá (Born y Forrest, 2019, p. 632), cuyas disposiciones autorizaban, desde 2004, tanto la presentación de amicus curiae, como la obligación de los tribunales arbitrales de admitirlos, bajo ciertos requisitos.

Esa nueva tendencia por incluir la participación de terceras partes no contendientes, en cierta medida, estuvo influenciada por la jurisprudencia del SSCIE: en un inicio, frente a la inexistencia de disposiciones expresas y específicas para aceptar escritos 
voluntarios de terceros ajenos a los AII como amicus curiae, los tribunales arbitrales decidieron admitirlos en base a su autonomía y discrecionalidad. Recordemos que esta práctica empezó con el caso CNUDMI Methanex c. Estados Unidos. Este tribunal arbitral —derivado del capítulo 11 del Tratado de Libre Comercio de América del Norte (TLCAN) y conducido por las Reglas de Arbitraje CNUDMI-, admitió las observaciones de una organización no gubernamental sin ánimo de lucro, bajo la figura de amicus curiae, y de acuerdo con el entonces artículo 15.1 de dicho Reglamento.

Ese tribunal consideró, en su Decisión sobre Peticiones de Terceras Personas para intervenir como Amicus Curiae, del 15 de enero de 2001, que la disputa mencionada iba más allá de la esfera privada de las partes, al verificar aspectos de interés público — como el medio ambiente y la salud—, motivo por el cual, decidió que el AII fuese más abierto y transparente $(\$ 49)$. Desde entonces, hubo un cambio en la poca atención brindada a los amicus curiae y su representación de los intereses públicos, siendo incorporados progresivamente en el SSCIE (Kent y Trinidad, 2016, p. 1092). Así lo demuestran los nuevos tratados de inversiones, con disposiciones sobre transparencia procesal. Pese a este favorable contexto, si bien se acepta cada vez más la participación de los amicus curiae, no cualquier tercero ajeno a los AII puede utilizar esta herramienta procesal.

Antes de analizar esa última problemática de los amicus curiae, en esta sección nos referiremos a los instrumentos internacionales que contienen disposiciones sobre su admisión, haciendo énfasis en los tratados suscritos por los Estados de América Latina y el Caribe.

\subsection{En los tratados de inversiones}

Desde 2001, y principalmente a partir de la suscripción de los primeros instrumentos internacionales de inversiones con cláusulas que permitan la participación de terceras partes no contendientes en los AII, el SSCIE ha sido testigo del incremento sustancial en la presentación de solicitudes de amicus curiae (Baltag, 2020, p. 4). Con este positivo desarrollo en favor de la credibilidad y legitimidad del SSCIE, de igual modo, se promueve que tanto actores no estatales como entidades públicas variadas representen los intereses públicos que consideren son deficientemente defendidos por los Estados o que pasan desapercibidos para los tribunales arbitrales.

Asimismo, esa creciente importancia del rol de los amicus curiae en el SSCIE, formalmente podemos observarla en una gran diversidad de preceptos descritos, por citar algunos ejemplos, en los siguientes los tratados bilaterales de inversión: 
i) Tratado relativo a la Promoción y Protección Recíproca de Inversiones entre Uruguay y Estados Unidos de América de 2005 (artículos 28 y 29): se dispone que las partes no contendientes podrán hacer comunicaciones orales o escritas sobre la interpretación de este tratado bilateral. Se aclara que tales partes personas o entidades - podrán presentar solicitudes de amicus curiae, dejando al criterio del tribunal arbitral aceptarlos y considerarlos. Las audiencias estarán abiertas al público. La información protegida no será de acceso al público y a las partes no contendientes.

ii) Tratado para la Promoción y Protección de Inversiones entre Canadá y Perú de 2006 (artículo 38 y 39): además de facilitar las audiencias públicas y toda documentación, previa consultas a las partes, cualquier parte no contendiente podrá remitir una solicitud de presentación de escrito a los tribunales arbitrales y a las partes. Igualmente, estos escritos tendrán que demostrar, entre otros, que sus aportes son diferentes a las argumentaciones de las partes, que tienen un interés significativo en la controversia, y que la discusión de esta también incorpora algún interés público.

iii) Tratado de Inversión entre Chile y Hong Kong de 2016 (artículo 26 y 27): se aceptarán y considerarán amicus curiae de partes no contendientes - personas o entidades- que puedan ayudar a los tribunales arbitrales en la evaluación de las presentaciones y argumentos de las partes. A su vez, este tratado permite que las partes no contendientes dirijan solicitudes a los tribunales arbitrales sobre cualquier cuestión relativa a la interpretación del tratado, y recibir copia de la información de los procedimientos arbitrales, inclusive las confidenciales.

\subsection{En los tratados comerciales con temas de inversión}

Desde la interpretación del Capítulo 11 del TLCAN de 1992 que hizo el tribunal arbitral en el caso CNUDMI Methanex, acerca de la admisión de terceras partes no contendientes en los procedimientos arbitrales, subsecuentes instrumentos internacionales con temas de inversión han sido adoptados, incorporando de manera expresa y tácita la presentación de amicus curiae y otras formas de participación. Para ejemplificar, este importante desarrollo de la transparencia en el AII, tenemos:

i) Tratados de Libre Comercio entre: Chile-Estados Unidos de América de 2003 (artículos 10.19 y 10.20); México-Panamá de 2004 (artículos 10.21 y 10.22); República Dominicana-Centroamérica (Costa Rica, El Salvador, Guatemala, Honduras, Nicaragua)-Estados Unidos de América de 2004 (artículos 10.20 y 10.21); Chile-Colombia de 2006 (artículos 9.20 y 9.21); Chile-Perú de 2006 (artículos 11.20 y 11.21); Nicaragua-Taiwán de 2006 (artículos 10.20 y 
10.21); Australia-Chile de 2008 (artículos 10.20 y 10.21); Canadá-Colombia de 2008 (artículos 830 y 831); Canadá-Perú de 2008 (artículos 835 y 836); Canadá-Panamá de 2010 (artículos 9.30 y 9.31); Costa Rica-Perú de 2011 (artículos 12.21 y 12.22); Guatemala y Perú de 2011 (artículos 12.21 y 12.22); México-Centroamérica de 2011 (artículos 11.24 y 11.25); CanadáHonduras de 2013 (artículos 10.35 y 10.36); Chile-Tailandia de 2013 (artículos 14.10 y anexo 14.10); Colombia-Costa Rica de 2013 (artículos 12.22 y 12.23); Honduras-Perú de 2015 (artículos 12.21 y 12.22); y, Perú-Australia de 2018 (artículos 8.24 y 8.25).

ii) Acuerdos de promoción comercial entre: Colombia-Estados Unidos de América de 2006 (artículos 10.20 y 10.21); Perú-Estados Unidos de América de 2006 (artículos 10.20 y 10.21); y, Estados Unidos de América-Panamá de 2007 (artículos 10.20 y 10.21).

iii) Acuerdo comercial entre la Unión Europea-Colombia-Perú de 2012, y su Protocolo de Adhesión para tener en cuenta a Ecuador de 2014 (artículo 316).

iv) Acuerdos de Asociación Económica entre: la Unión Europea-el Foro del Caribe (CARIFORUM) de 2008 (artículo 217), y CARIFORUM-Reino Unido de 2019 (artículo 217).

v) Acuerdo Marco de la Alianza del Pacífico y su Protocolo Adicional de 2014 (artículos 10.20 y 10.21).

\subsection{En otros instrumentos internacionales}

La Convención de Mauricio sobre Transparencia de 2014, que refuerza la participación de terceras partes no contendientes y la conducción de los AII bajo ciertas condiciones, adicionalmente ha logrado que los Estados se muestren más receptivos y abiertos en la búsqueda de una mayor transparencia en el SSCIE. Recientemente, y en concordancia con los estándares proporcionados por dicha Convención, un creciente número de Estados —además de los señalados en los subpuntos anteriores- han suscrito una nueva generación de tratados de inversión que regulan tanto la presentación de solicitudes de amicus curiae, como otras formas de participación.

La voluntad de los Estados por lograr mayor legitimidad y credibilidad en el SSCIE, también la encontramos en los siguientes instrumentos internacionales: el Acuerdo de Colaboración y Cooperación Reforzadas entre la Unión Europea y Kazajistán de 2015 (artículo 193); el Acuerdo de Asociación Económica entre la Unión Europea y la Comunidad de Desarrollo de África Austral de 2016 (artículo 90); el Acuerdo Económico y Comercial Global de 2016 (artículos 8.36 y 8.38); el Acuerdo de 
Asociación Global y Reforzado entre la Unión Europea y Armenia de 2017 (artículo 336); el Acuerdo entre la Unión Europea y Japón relativo a una Asociación Económica de 2018 (artículo 21.17); el Acuerdo de Protección de Inversiones entre la Unión Europea y Singapur de 2018 (artículos 3.16 y 3.17); el Tratado entre México, Estados Unidos de América y Canadá de 2018 (artículos 14.D.7 y 14.D.8); el Tratado Integral y Progresista de Asociación Transpacífico de 2018 (artículos 9.23 y 9.24); y, el Acuerdo de Protección de Inversiones entre la Unión Europea y Vietnam de 2019 (artículos 3.46 y 3.51).

Cabe señalar que, de los ejemplos descritos resaltan los modelos de tratados de inversión de la Unión Europea, porque además de admitir expresamente los amicus curiae o tácitamente el Reglamento CNUDMI sobre Transparencia —como así lo refieren las disposiciones de dichos modelos-, establecen un tribunal permanente de inversiones y un tribunal permanente de apelación para solucionar los conflictos de inversión. Tales figuras componen el nuevo sistema bilateral de tribunales de inversión, que es parte de un futuro mecanismo permanente, y eventualmente, reemplazaría el uso del SSCIE. Se encuentra regulado en el Acuerdo Económico y Comercial Global de 2016, en el Acuerdo de Protección de Inversiones entre la Unión Europea y Singapur de 2018, y en el Acuerdo de Protección de Inversiones entre la Unión Europea y Vietnam de 2019.

\subsection{En los actuales estudios del Grupo de Trabajo III de la CNUDMI sobre la Reforma del Sistema de Solución de Controversias entre Inversionistas y Estados}

Después de evaluar las Notas de la Secretaría de la CNUDMI acerca de la Posible Labor Futura sobre los procesos paralelos y la ética en los AII, y de la reforma del SSCIE, en 2017 la CNUDMI encargó a su Grupo de Trabajo III que estudiara y desarrollara diversas soluciones de reforma (CNUDMI, 2017, \$ 264). Discrecionalmente, este Grupo optó por dividir su trabajo en tres fases: determinar y analizar los problemas; discutir el inicio de la reforma; y, de ser esta última deseable, recibir propuestas para la reforma del SSCIE, las cuales servirán para elaborar una recomendación final que deberá ser presentada a la CNUDMI. En la actualidad, y a pesar de las dificultades, el Grupo de Trabajo III se encuentra cumpliendo el mandato en su última fase.

Respecto a la garantía de la participación de partes no contendientes o terceros interesados en los procedimientos arbitrales -mediante escritos-, el Grupo de Trabajo III la examinó en 2019. Se discutió, por un lado, que dicha participación clasificaba en la categoría de otras inquietudes de aspectos procesales que debían discutirse para una reforma final del SSCIE, y por otro lado, que incluía al "público en general 
y la comunidad local afectada por la inversión o la controversia de que se tratara» (CNUDMI, 2019, \$31). Además, se cuestionó si algunos aspectos del Reglamento CNUDMI sobre Transparencia y del Convenio de Mauricio sobre Transparencia, respecto a la presentación de amicus curiae, eran insuficientes, y si era factible mejorarlos desde otra perspectiva: primero instruyendo a los tribunales arbitrales mediante orientaciones (CNUDMI, 2019, $\$ 32$ ). Se creyó prudente que estas interrogantes fuesen resueltas cuando el Grupo de Trabajo III tratara, tanto los problemas de la falta de concordancia y de precisión de los laudos, como la elaboración de medidas para «dar a las partes en el tratado un mayor control sobre el proceso» (CNUDMI, 2019, §33) del SSCIE.

\section{El tratamiento de los amicus curiae en los arbitrajes internacionales de inversión}

Si bien los instrumentos internacionales mencionados autorizan la introducción de terceras partes no contendientes en los AII, de igual modo los amicus curiae son regulados en ciertos Reglamentos de Arbitrajes tanto de los mecanismos más frecuentemente utilizados - CIADI y CNUDMI — como en otros. Seguidamente, pasaremos a examinar cuáles son las condiciones de esos mecanismos para que los tribunales arbitrales acepten los escritos de amicus curiae.

\subsection{En los procedimientos arbitrales de inversión conforme al mecanismo CIADI}

Antes de las modificaciones del Convenio CIADI, y de sus reglas y reglamentos en 2006, los tribunales arbitrales regidos por este mecanismo, consideraban la admisión de los amicus curiae, sobre la base de su discrecionalidad y poder de decisión frente a vacíos procedimentales - Artículo 44 del Convenio CIADI- Ahora con disposiciones expresas, como la Regla CIADI 37(2), y el artículo 41(3) del Reglamento de Arbitraje del Mecanismo Complementario CIADI, se señala que los tribunales arbitrales admitirán los amicus curiae, después de consultar a las partes, y teniendo en cuenta que no alteren los arbitrajes ni los recarguen indebidamente. Tampoco deberán perjudicar injustamente a las partes, aunque estas podrán presentar observaciones respecto a los amicus curiae.

Del mismo modo, los amicus curiae serán admitidos siempre y cuando:

i) ayuden a los tribunales arbitrales a identificar cuestiones de hecho o de derecho relacionadas con el arbitraje, y aporten perspectivas, conocimientos o visiones distintos a los presentados por las partes;

ii) se refieran a una cuestión relativa a la disputa; $y$, 
iii) quienes presenten las solicitudes, tengan intereses significativos en las controversias, y a su vez demuestren independencia de las partes.

\subsection{En los procedimientos arbitrales de inversión ad hoc conforme al mecanismo CNUDMI}

A diferencia del mecanismo CIADI, las Reglas de Arbitraje CNUDMI no fueron modificadas para adecuarse a la nueva tendencia de transparentar los AII, sino complementadas con el Reglamento CNUDMI sobre Transparencia, disposición procedimental que autoriza a los tribunales arbitrales a recibir y aceptar escritos de amicus curiae. Este último Reglamento menciona que, tras consultar con las partes, los tribunales arbitrales ad hoc CNUDMI, o arbitrajes institucionales que estén conducidas por las Reglas de Arbitraje CNUDMI, permitirán que terceras partes no contendientes presenten solicitudes de amicus curiae si estos últimos guardan relación con los objetos de las controversias (artículo 4.1). Asimismo, por escrito, estas terceras partes deberán declarar sus identidades, cuáles son sus intereses en los arbitrajes, si tienen alguna filiación directa o indirecta con las partes contendientes, si han recibido algún tipo de ayuda - especialmente financiera en los dos últimos años-, y cuáles son las cuestiones concretas de hecho y de derecho de las disputas que deseen abordar (artículo 4.2).

Para que los amicus curiae sean admitidos, los tribunales arbitrales CNUDMI deben tener en consideración si dichas terceras partes tienen «un interés considerable» (artículo 4.2.a) y que sus participaciones aporten «enfoques, conocimientos particulares o puntos de vista distintos a los de las partes litigantes» (artículo 4.2.b). De igual forma, los tribunales arbitrales CNDUMI asegurarán que los amicus curiae no afecten indebidamente a los procedimientos arbitrales ni a las partes contendientes (artículo 4.5), concediendo a estas «una oportunidad razonable de formular observaciones» sobre los mismos (artículo 4.6).

\subsection{En los procesos arbitrales de inversión conforme a otros mecanismos}

Como es sabido, además de los dos anteriores principales mecanismos con los que se llevan adelante los procedimientos arbitrales entre inversionistas y Estados, existen otros que también arbitran conflictos de inversión, y cuyos Reglamentos de Arbitraje, indirectamente, posibilitan la intervención de terceras partes no contendientes a través de los escritos de amicus curiae. Bajo la idea que hemos planteado sobre la representación de los intereses públicos, la mayoría de estos mecanismos hacen viable que esas terceras partes participen en los AII. Así, tenemos los siguientes: 
i) la Corte Permanente de Arbitraje: el Reglamento de Arbitraje de 2012, permite que a instancia de cualquier parte, los tribunales arbitrales autoricen la participación de terceros invitados, siempre que sean parte del acuerdo de arbitraje (artículo 17.5). Adicionalmente, estos tribunales podrían aceptar escritos de amicus curiae, previa consulta a las partes, ya que tienen la facultad de conducir los arbitrajes de manera apropiada a fin de llegar a «una solución justa y eficaz del litigio entre las partes» (artículo 17.1).

ii) el Instituto de Arbitraje de la Cámara de Comercio de Estocolmo: el artículo 3 del Apéndice III del Reglamento de Arbitraje de 2020 indica que podrán presentar solicitudes cualquier persona que no sea parte litigante ni parte del tratado (artículo 3.1), bajo ciertos requisitos. Por ejemplo, las solicitudes de amicus curiae deberán describir la identificación del solicitante, tanto su interés en el AII como las cuestiones concretas de hecho o de derecho de los mismos, su afiliación directa o indirecta con las partes contendientes, y si han recibido alguna ayuda directa o indirectamente para el diseño de la solicitud (artículo 3.2). Además, de otras situaciones importantes, para admitir los amicus curiae, los tribunales arbitrales tendrán que consultar a las partes y deberán valorar la índole y relevancia de dicho interés, y si las cuestiones planteadas aportan «una perspectiva, conocimientos particulares o puntos de vista distintos a los de las partes...» (artículo 3.3). Los tribunales arbitrales concederán a las partes contendientes la oportunidad de formular observaciones a las solicitudes (artículo 3.8), y asegurarán que estas solicitudes no dificulten los procedimientos arbitrales ni perjudiquen indebidamente a las partes en el proceso (artículo 3.9).

iii) el Centro Internacional de Arbitraje de Viena: el Reglamento de Arbitraje de 2018 señala que, previa autorización de las partes contendientes, los tribunales arbitrales decidirán el ingreso de terceros en sus procedimientos (artículo 14.1). Conforme a una de las opiniones del Grupo de Trabajo que revisó este reglamento, se entiende que el término terceros comprende cualquier forma de participación, inclusive las presentaciones de amicus curiae (Zuberbühler, Müller y Habegger, 2012, p. 253).

\section{La protección de la salud pública en los arbitrajes internacionales de inversión}

Para que el inversionista extranjero afectado negativamente por alguna regulación estatal pueda recurrir al AII, debe demostrar que su inversión califica dentro de la definición descrita en el tratado de inversión aplicable. Asimismo, si el convenio 
arbitral establece que en caso de controversia el arbitraje se regirá por el reglamento arbitral del CIADI, para un sector de la doctrina y de la jurisprudencia del SSCIE, la inversión estará cubierta en la medida en que cumpla con ciertos criterios adicionales referidos al concepto de inversión. La Decisión sobre Jurisdicción del 31 de julio de 2001, del caso CIADI Salini Costruttori S.p.A. y Italstrade S.p.A. (I) c. Marruecos (\$ 52), introduce el test Salini y adopta los cuatro criterios que hasta ese momento en la doctrina se venían discutiendo para definir la inversión. Uno de esos criterios señala que la inversión debe contribuir al desarrollo económico del Estado receptor de la inversión. Sin embargo, aun cuando en la ejecución de la inversión puedan haberse ocasionado daños a intereses públicos, ello no justifica desconocer dicha inversión como tal (Sheargold y Mitchell, 2019, p. 5).

Más allá de resolver si el Estado demandado incumplió o no las obligaciones del tratado de inversión pertinente, con el fin de brindar una solución integral — sin implicaciones para terceros-, los tribunales arbitrales deben reconocer la existencia de intereses o derechos que podrían ser prioritarios y que requieren de protección especial, como la salud pública. Por ello, resulta necesario que estos tribunales acepten las opiniones de expertos, que colaborarán en la comprensión de los intereses públicos involucrados, precisamente porque los integrantes de los tribunales arbitrales no son necesariamente expertos en todos los campos académicos, especialmente en áreas como el derecho internacional de la salud pública y en el manejo de los conflictos de normas con otros regímenes del derecho internacional.

\subsection{El derecho internacional de la salud pública}

El derecho internacional de la salud, actualmente denominado derecho global de la salud, porque incluye en su estructura a la comunidad internacional en general, es un régimen del derecho internacional relativamente nuevo y que, antes de la pandemia COVID-19, generaba poca atención dentro del arbitraje de inversiones. Su ordenamiento jurídico especial abarca elementos de otros regímenes jurídicos internacionales - derecho internacional de los derechos Humanos, derecho internacional ambiental, derecho internacional económico, derecho internacional humanitario-, relacionados con la protección esencial de la salud pública, con el fin de garantizar un acceso equitativo y global, lo cual se traduce en la posibilidad de que la población mundial cuente con el más alto estándar de salud física y mental (Gostin, 2014, p. 59). Respecto al concepto de salud, cabe decir que presenta ambigüedades y no existe una clara definición (Vadi, 2014, pp. 28-29), muestra de ello son los conceptos desarrollados por la Organización Mundial de la Salud (OMS) y el Comité de Derechos Económicos, Sociales y Culturales de Naciones Unidas. En el primer caso, la salud es concebida como «un estado de completo bienestar físico, mental y social, y no 
solamente la ausencia de afecciones o enfermedades» (Preámbulo de la Constitución de la OMS). En el segundo caso, la salud está formulada como:

[...] un derecho humano fundamental e indispensable para el ejercicio de los demás derechos humanos. Todo ser humano tiene derecho al disfrute del más alto nivel posible de salud que le permita vivir dignamente. La efectividad del derecho a la salud se puede alcanzar mediante numerosos procedimientos complementarios, como la formulación de políticas en materia de salud, la aplicación de los programas de salud elaborados por la Organización Mundial de la Salud (OMS) o la adopción de instrumentos jurídicos concretos. Además, el derecho a la salud abarca determinados componentes aplicables en virtud de la ley. (Observación General No 14, 2000, \$1)

Otros instrumentos internacionales relevantes que también contemplan el derecho a la salud, son, por ejemplo: la Declaración Universal de Derechos Humanos (artículo 25); la Carta de las Naciones Unidas (artículo 55); el Pacto Internacional de Derechos Económicos, Sociales y Culturales (artículo 12); la Declaración Americana de Derechos y Deberes del Hombre (artículo XI); la Convención Americana sobre Derechos Humanos (artículo 26); y, el Protocolo Adicional a la Convención Americana de Derechos Humanos en materia de Derechos Económicos, Sociales y Culturales (artículo 10).

En cuanto a la jurisprudencia del sistema regional de derechos humanos, en la sentencia del 8 de marzo de 2018 del caso Poblete Vilches y otros c. Chile Fondo, Reparaciones y Costas-, la Corte Interamericana considera que la salud «es un derecho humano fundamental e indispensable para el ejercicio adecuado de los demás derechos humanos» ( $\$ 118)$. Además menciona que los Estados tienen tanto el deber de garantizar «el acceso de las personas a servicios esenciales de salud» $(\$ 118)$ como «regular con carácter permanente la prestación de servicios... y la ejecución de programas nacionales relativos al logro de una prestación de servicios de calidad» (\$119).

En relación con la crisis sanitaria causada por la pandemia COVID-19, la OMS tiene un importante rol, puesto que es «la autoridad directiva y coordinadora en asuntos de sanidad internacional» (artículo 2.a. de la Constitución de la OMS). Como primera agencia especializada de Naciones Unidas, desde 1948 la Asamblea Mundial de la Salud ha adoptado diversos instrumentos internacionales sobre salud pública, como el Reglamento Sanitario Internacional de 2005 (RSI): uno de los acuerdos vinculantes más importantes que tiene como finalidad garantizar la prevención, la protección y el control de la propagación internacional de enfermedades, mediante una respuesta de salud pública adecuada y que mínimamente interfiera con el tráfico y el comercio mundial (artículo 2 RSI). Para que los Estados Partes adopten medidas de contención contra el brote de la coronavirus de tipo 2 causante del síndrome respiratorio 
agudo severo, conforme con las disposiciones del RSI, el director de la OMS Tedros Ghebreyesus determinó —el 30 de enero de 2020— que tal brote constituía una emergencia de salud pública de importancia internacional (artículo $12 \mathrm{RSI}$ ), por lo que formuló recomendaciones temporales con el objetivo que los Estados Partes (artículo 15 RSI) redujeran la infección y la propagación internacional.

Tales recomendaciones, en su momento, no fueron percibidas por los Estados Partes como la mejor solución para contrarrestar el impacto de la COVID-19. De allí que, en base a otras obligaciones internacionales sobre derechos humanos, como las contenidas en el Pacto Internacional de Derechos Económicos, Sociales y Culturales, tales Estados aplicaron medidas sanitarias, distintas a las señaladas en el RSI, que restringían el tráfico internacional y comercial, para proteger urgente y equitativamente el derecho a la salud de sus poblaciones, y evitando con ello directa o indirectamente poner en riesgo la salud pública. Con respecto al AII, es probable que la relación entre esas medidas estatales y las obligaciones vinculantes de los tratados de salud pública sea mejor explicada por los amicus curiae, en los AII que muy probablemente se iniciarán, coadyuvando en el conocimiento técnico del caso por parte de los tribunales arbitrales.

\subsection{La colisión entre los tratados de inversión y los tratados de salud pública}

El posible enfrentamiento entre el derecho internacional de las inversiones y el derecho global de la aalud que se produzca en los AII debe tener una evaluación específica en cada caso, aun cuando los diferentes intereses que se busquen proteger, es decir la salud y la propiedad privada, sean de derechos humanos. Independientemente de ello, los tribunales arbitrales deben considerar la jurisprudencia del SSCIE, que reconoce el derecho de los Estados a regular los intereses públicos, siempre que las medidas a implementarse sean promulgadas de buena fe y en forma no arbitraria o discriminatoria. Por tanto, los argumentos que empleen para justificar sus decisiones no solo deben ser coherentes y razonables, también deben demostrar concordancia con el derecho internacional y los derechos humanos, a pesar de que los tratados de inversión, en general, mantengan silencio sobre la interacción con otros regímenes de derecho internacional (De Brabandere, 2019, p. 638).

En ese sentido, los árbitros tienen la obligación de interpretar adecuadamente tales regímenes jurídicos internacionales de acuerdo con el artículo 31.3c) de la Convención de Viena sobre el Derecho de los Tratados, más aún cuando en la contextualización de las disputas se percibe la presencia de normas de ius cogens. Para aclarar y equilibrar la relación entre el derecho internacional de las inversiones y el derecho internacional de la salud pública, sostenemos que si bien queda a la discrecionalidad de los tribunales arbitrales determinar cómo resuelven las controversias particulares, 
ellos no tendrían que interpretar estos ordenamientos por separado, a pesar que sean regímenes jurídicos autónomos, o solo aplicar la norma que disponga el tratado de inversión pertinente, puesto que resulta relevante que los operadores internacionales logren, ante todo, la unidad del derecho internacional (Vadi, 2014, p. 87). En consecuencia, las acciones de salud pública que los Estados hayan adoptado para enfrentar la propagación de la pandemia COVID-19 y su adecuación con el ejercicio legítimo de facultades regulatorias — siempre y cuando no sean acciones arbitrarias y/o discriminatorias contra los derechos de los inversionistas-, es un asunto que debe ser examinado cuidadosamente por los tribunales arbitrales y no ser ignorado al momento de laudar (Sheargold y Mitchell, 2019, p. 13).

Queda claro que las interacciones problemáticas entre el derecho internacional de las inversiones, y el derecho internacional de la salud pública, en los próximos AII, serán más profundas y complejas si los tribunales arbitrales aplican, sin un análisis integral, la lex speciallis del derecho internacional de inversiones en la resolución de los conflictos. Es indispensable que ellos consideren que a causa de la pandemia COVID-19, la defensa de los Estados demandados estará más enfocada en justificar la apropiada imposición de medidas para salvaguardar la salud pública, descartando o atenuando con ello la responsabilidad estatal por supuestos incumplimientos de los respectivos tratados de inversión. Por ello, sostenemos que en estas circunstancias se hace indispensable conocer otras perspectivas e informaciones técnicas - como las que puedan proporcionar los amicus curiae - que amplíen la visión de los tribunales arbitrales y que coadyuven en la emisión de decisiones justas y razonables, fortaleciendo con ello la legitimidad del SSCIE.

\subsection{Los amicus curiae sobre salud pública en los arbitrajes internacionales de inversión}

Hasta el momento, en el SSCIE existen tres tribunales arbitrales que aceptaron amicus curiae, cuyos arbitrajes discutieron expresamente que las medidas estatales impugnadas por los inversionistas demandantes fueron adoptadas para proteger directamente la salud pública. Tales amicus curiae plantearon diferentes consideraciones que permitieron, de un lado, el inicio y el posterior desarrollo de la transparencia en el SSCIE, y del otro lado, reconocer la importancia de la aplicación de otros regímenes del derecho internacional para resolver los conflictos entre inversores y Estados de manera razonable y justa. A continuación, describiremos los casos con implicaciones de salud pública, en que se presentaron estos amicus curiae tan significativos.

En el ya mencionado caso CNUDMI Methanex se discutió la regulación del éter metil tert-butílico (MTBE siglas en inglés), un compuesto químico producido a partir del isobutileno y metanol, y que se utiliza como aditivo de la gasolina que 
mejora su resistencia a la combustión incontrolada, y su oxigenación. En 1999, el estado de California ordenó eliminar gradualmente el MTBE, basado en un estudio sobre los efectos negativos de posibles contaminaciones en aguas subterráneas y para la salud pública, prohibiendo la venta y el uso de gasolina producida con MTBE a partir de 2003. Ese mismo año, Methanex, un productor y comercializador canadiense de metanol, inició el AII alegando que tal medida regulatoria violaba el nivel mínimo de trato y la no expropiación. Durante el procedimiento arbitral, tanto el Instituto de Desarrollo Sostenible Internacional, como Communities for a Better Environment, Bluewater Network of Earth Island Institute y el Centro de Derecho Ambiental Internacional solicitaron permiso al tribunal arbitral para presentar escritos de amicus curiae. Conforme con el entonces artículo 15.1 del Reglamento de Arbitraje CNUDMI, el tribunal arbitral estableció que tenía el poder de aceptar los amicus curiae, sosteniendo que proporcionaban respaldo al procedimiento arbitral y eran apropiados para asistirlos por el indudable interés público del caso Methanex (Decisión del Tribunal sobre Peticiones de Terceras Personas para intervenir como amicus curiae del 15 de enero de 2001, $\$ 47-49$ ).

De este modo, este tribunal arbitral pasó a ser el primer tribunal establecido bajo el Capítulo 11 del TLCAN y conducido por las Reglas de Arbitraje CNUDMI que aceptó amicus curiae. Las opiniones proporcionadas por estas terceras partes no contendientes fueron muy similares a los argumentos descritos por el tribunal arbitral en el Laudo Arbitral sobre Jurisdicción y Méritos del 3 de agosto de 2005 y que están referidas a la implicancia del interés público en el AII, señalando que la medida regular cuestionada fue legal, con un propósito público, no fue discriminatoria y se realizó dentro del debido proceso (Parte IV, Capítulo D, $\$ 15$ ).

Por su parte, en el caso CIADI Philip Morris Brand Sàrl, Philip Morris Products y Abal Hermanos S.A. c. Uruguay, se interpusieron con éxito dos solicitudes de amicus curiae: el primero pertenecía a la OMS conjuntamente con la Secretaría del Convenio Marco para el Control del Tabaco, y el segundo era de la Organización Panamericana de la Salud. Ambos fueron aceptados por el tribunal arbitral mediante la Orden Procesal $N^{\circ} 3$ del 17 de febrero de 2015, y la Orden Procesal $N^{\circ} 4$ de marzo de 2015, respectivamente, ya que según este tribunal podrían ser beneficiosos en el proceso de la toma de decisiones. También se presentaron dos solicitudes que fueron rechazadas y que pertenecían a la Fundación Avaaz, y a la Asociación Interamericana de la Propiedad Intelectual. El caso se inició contra Uruguay en 2010 por sus regulaciones sobre la prohibición de comercializar más de una variante de cigarrillos bajo una misma familia de marca en 2008, y las advertencias sanitarias en los etiquetados de los empaques del tabaco en 2009, medidas que fueron promulgadas en concordancia con las obligaciones del mencionado Convenio Marco. Los inversionistas deman- 
dantes sostuvieron que tales regulaciones constituían violaciones de las obligaciones de Uruguay por el Tratado Bilateral de Inversiones Suiza-Uruguay — menoscabo del uso y goce de las inversiones, tratamiento justo y equitativo y denegación de justicia, expropiación y observancia de los compromisos-.

En el laudo arbitral del 8 de julio de 2016 de este caso CIADI, el tribunal arbitral empleó y citó la información de los amicus curiae en su análisis jurídico. Ello le permitió declarar que las regulaciones de control del tabaco fueron diseñadas de buena fe con el propósito de proteger la salud pública uruguaya ( $\$ 306,409,420)$. Por tanto, las medidas no constituyeron una expropiación $(\$ 307)$, no fueron arbitrarias, inequitativas, injustas, discriminatorias o desproporcionales ( $\$ 410$ y 420 ), tampoco fueron obstáculos injustificados a las inversiones ( $\$ 445)$, no existieron compromisos adquiridos $(\$ 458,482)$ y no existió denegación de justicia $(\$ 536,580)$. Para el tribunal, la protección de la salud pública era un ejercicio legal del poder policía de Uruguay, una manifestación esencial reconocida mucho antes que "en las decisiones en materia de tratados de inversión» (\$292). De igual forma, reconoció que:

[...] la responsabilidad por las medidas de salud pública recae sobre el gobierno y los tribunales constituidos en casos de inversión deberían prestar gran deferencia a los criterios gubernamentales, respecto de las necesidades nacionales en cuestiones como la protección de la salud pública. En tales casos se debe respeto al «ejercicio discrecional del poder soberano que no se lleve a cabo de manera irracional o de mala fe $[\ldots] . »(\$ 399)$

En el caso CIADI Eli Lilly y Compañía c. Canadá, la empresa farmacéutica inició el procedimiento arbitral en 2012 alegando que la revocación de las patentes que protegían sus medicamentos Strattera y Zyprexa por tribunales federales canadienses en 2010 y 2011 - ya que no demostró la utilidad prometida de los productos al momento de patentarlos-, la consideraba una expropiación ilegal de su propiedad intelectual y una conducta violatoria del nivel mínimo de trato.

De los seis amicus curiae presentados durante este procedimiento, el tribunal arbitral, mediante Orden Procesal N 4 del 23 de febrero de 2016, aceptó cinco en su integridad -Canadian Chamber of Commerce; Canadian Generic Pharmaceutical Association; Samuelson-Glushko Canadian Internet Policy \& Public Interest Clinic, and the Centre for Intellectual Property Policy; Intellectual property law professors from universities in the United States; y, National Association of Manufacturers—, uno parcialmente — A group of academics from the US, UK, Switzerland, South Africa and Nepal con respecto de doctor. Burcu Kilic, profesor Brook K. Baker, profesor Cynthia Ho y Yaniv Heled J.S.D—, y rechazó tres - Three professors from the University of Cambridge; Innovative Medicines Canada and BIOTECanada; y, Pharmaceutical Research and Manufacturers of America, Mexican Association of the Research Based Pharmaceutical Industry and Biotechnology 
Innovation Organization-. Precisamos que de los seis amicus curiae aceptados, expresamente dos mencionaban que en esta disputa se encontraba involucrado el interés público de la salud pública, en concreto, el derecho a un mejor acceso a medicamentos para todos - Intellectual property law professors from universities in the United States; y $A$ group of academics from the US, UK, Switzerland, South Africa and Nepal- En el laudo arbitral del 16 de marzo de 2017, este tribunal resolvió que Canadá no incumplía ninguna obligación del TLCAN, rechazando con ello la demanda (\$442).

\section{Impacto de la pandemia COVID-19 y relevancia de los amicus curiae en controversias sobre salud pública - Reflexiones finales}

En este grave contexto sanitario sin precedentes, sostenemos que los amicus curiae al enfocarse en dar voz a los intereses públicos y contribuir en la recuperación de la legitimidad del SSCIE, serán determinantes en los resultados de los próximos arbitrajes que se inicien por las medidas estatales adoptadas para frenar la propagación del virus COVID-19. Aunque en la mayoría de los casos, probablemente, la adopción de medidas preventivas para proteger la salud pública se hizo de buena fe y de manera razonable, los demandantes inversionistas, en muchos casos, no compartirán esta posición, argumentando que las medidas no respetaron los estándares básicos de protección a la inversión que ofrecen los tratados de inversión.

Evidentemente, este enfrentamiento respecto a qué intereses deben ser protegidos de manera prioritaria en una situación de emergencia, sea la salud pública o las inversiones extranjeras, en los arbitrajes internacionales de inversión y cuáles son los límites, impuestos por el derecho internacional, que deben ser respetados por los Estados, incluso en situaciones excepcionales de emergencia, siempre será concebido como un gran desafío para los tribunales arbitrales. Desde esta perspectiva, consideramos que los amicus curiae serán un elemento de apoyo fundamental para esclarecer los fundamentos del ejercicio de las facultades regulatorias de los Estados y con ello, poder determinar su legitimidad o arbitrariedad.

Creemos que, tal y como sucedió en el caso Philip Morris c. Uruguay, en los próximos AII los amicus curiae no solo serán aceptados, sino también citados y aplicados con mayor frecuencia en el análisis de los tribunales arbitrales para confirmar la legalidad de las medidas de salud pública impuestas por los Estados durante la pandemia COVID-19. Ello no solo implica que los tribunales arbitrales empiecen a reconocer los impactos específicos de los amicus curiae, lo cual aún no ha sucedido (Baltag, 2020, p. 29), sino que también involucra el desarrollo de una relación de apoyo real y transparente de los amicus curiae frente a los tribunales arbitrales. 


\section{Referencias bibliográficas}

Baltag, C. (2020). The Role of Amici Curiae in Light of Recent Developments in Investment Treaty Arbitration: Legitimizing the System? ICSID Review, 1-32. https://doi. org/10.1093/icsidreview/siaa021

Born, G. y Forrest, S. (2019). Amicus Curiae Participation in Investment Arbitration. ICSID Review, 34(3), 626-665. https://doi.org/10.1093/icsidreview/siz020

Butler, N. (2019). Non-Disputing Party Participation in ICSID Disputes: Faux Amici? Netherlands International Law Review, 66, 143-178. https://doi.org/10.1007/ s40802-019-00132-8

Comisión de las Naciones Unidas para el Derecho Mercantil Internacional. (2017). Posible reforma del sistema de solución de controversias entre inversionistas y Estados. Secretaría de la CNUDMI.

Comisión de las Naciones Unidas para el Derecho Mercantil Internacional. (2019). Informe del Grupo de Trabajo III (Reforma del Sistema de Solución de Controversias entre Inversionistas y Estados) sobre la labor realizada en su $37^{\circ}$ periodo de sesiones. Secretaría de la CNUDMI.

De Brabandere, E. (2019). Human rights and international investment law. En M. Krajewski y R. T. Hoffmann (Ed.), Research Handbook on Foreign Direct Investment (pp. 619-645). Edward Elgar Publishing Limited.

De Brabandere, E. (2020). The (Ir)relevance of Transnational Public Policy in Investment Treaty Arbitration - A Reply to Jean-Michel Marcoux. Journal of World Investment \& Trade, (21), 847-866. https://doi.org/10.1163/22119000-12340196

Gostin, L. O. (2014). Global Health Law. Harvard University Press.

Kent, A. y Trinidad, J. (2016). International Law Scholars as Amici Curiae: An Emerging Dialogue (of the Deaf)? Leiden Journal of International Law, (29), 1081-1101. https:// doi.org/10.1017/S0922156516000510

Maupy, J. (2013). Transparency in International Investment Law: The Good, the Bad and the Murky. En A. Bianchi y A. Peters (Eds.), Transparency in International Law (pp. 142-171). Cambridge University Express.

Nater-Bass, G. y Rouvinez, C. (2013) Further Written Statements. En T. Zuberbühler, Ch. Müller y Ph. Habegger (Eds.), Swiss Rules of International Arbitration - Commentary (2a. Ed., pp. 248-253). Schulthess Juris.

Saei, J. (2017). Amicus curious: structure and play in investment arbitration, Transnational Legal Theory, 8(3), 247-295. https://doi.org/10.1080/20414005.2017.1385246

Sheargold, E. y Mitchell, A. (2019). Public Health in Investment Law and Arbitration. En J. Chaisse, L. Choukroune y S. Jusoh (Eds.), Handbook of International Investment Law and Policy. https://ssrn.com/abstract=3399749

\section{Jurisprudencia, normativa y otros documentos legales}

Constitución de la Organización Mundial de la Salud.

Convención de las Naciones Unidas sobre la Transparencia en los Arbitrajes entre Inversionistas y Estados en el Marco de un Tratado de 2014. 
Convención de Viena sobre el Derecho de los Tratados de 1969.

Convención sobre el Reconocimiento y la Ejecución de las Sentencias Arbitrales Extranjeras de 1958.

Convenio sobre Arreglo de Diferencias Relativas a Inversiones entre Estados y Nacionales de Otros Estados.

Decisión del Tribunal sobre Petición de Terceras Personas para intervenir como Amicus Curiae del 15 de enero de 2001 en el caso CNUDMI Methanex Corporation c. Estados Unidos de América.

Decisión del Tribunal sobre Petición de Terceras Personas para intervenir como Amicus Curiae del 17 de octubre de 2001 en el caso CIADI United Parcel Service of America Inc. (UPS) c. Canadá.

Decisión sobre Jurisdicción del 31 de julio de 2001en el caso CIADI Salini Costruttori S.p.A. y Italstrade S.p.A. (I) c. Marruecos.

Laudo Arbitral del 8 de julio de 2016 del caso CIADI Philip Morris Brand Sàrl, Philip Morris Products y Abal Hermanos S.A. c. Uruguay.

Laudo Arbitral del 16 de marzo de 2017 del caso CIADI Eli Lilly y Compañía c. Canadá.

Laudo Arbitral sobre Jurisdicción y Méritos del 3 de agosto de 2005 del caso CNUDMI Methanex Corporation c. Estados Unidos de América.

Observación General No 14 del Comité de Derechos Económicos, Sociales y Culturales. El derecho al disfrute del más alto nivel posible de salud (artículo 12 del Pacto Internacional de Derechos Económicos, Sociales y Culturales).

Orden en Respuesta a una Petición de Cinco Organizaciones No Gubernamentales de Permiso para hacer una Presentación de Amicus Curiae del 12 de febrero de 2007 en el caso CIADI Suez, Sociedad General de Aguas de Barcelona, S.A. y Vivendi Universal, S.A. c. Argentina.

Reglamento de la CNUDMI sobre la Transparencia en los Arbitrajes entre Inversionistas y Estados en el Marco de un Tratado de 2013.

Reglamento Sanitario Internacional de 2005.

Reglas de Arbitraje CIADI.

Reglas de Arbitraje CNUDMI.

Sentencia del 8 de marzo de 2018 de la Corte Interamericana de Derechos Humanos sobre el caso Poblete Vilches y otros c. Chile.

Tratado de Inversión entre Chile y Hong Kong de 2016.

Tratado para la Promoción y Protección de Inversiones entre Canadá y Perú de 2006.

Tratado relativo a la Promoción y Protección Recíproca de Inversiones entre Uruguay y Estados Unidos de América de 2005.

Fecha de recepción: 25 de abril de 2021 Fecha de aprobación: 20 de agosto de 2021 